\title{
Cycloadditionen mit Azosulfonen und Sulfonyliminen
}

Von Doz. Dr. F. Effenberger und Dipl.-Chem. R. Maier

Institut für Organische Chemie der Technischen Hochschule Stuttgart

Keten- $\mathrm{N}, \mathrm{N}$-acetale (1) und Keten- $\mathrm{O}, \mathrm{N}$-acetale (2) mit $\beta$ ständigem Wasserstoffatom geben mit Verbindungen, die ein elektrophiles Doppelbindungssystem enthalten, z. B. Isocyanaten oder Isothiocyanaten, $\beta$-Substitutionsprodukte [1, 2].

Azosulfone (3) ${ }^{[3]}$ und N-Sulfonylimine (7) ${ }^{[4]}$ reagieren dagegen mit Keten- $\mathrm{N}, \mathrm{N}$-acetalen (1) unter Cycloaddition $\mathrm{zu}$ den bisher noch nicht bekannten $\Delta^{3}-1,2$-Diazetinen (5) (Tabelle 1) bzw. 2-Azetinen (9) (Tabelle 2). Keten-O,N-acetale (2) geben mit (3) unter vergleichbaren Bedingungen ebenfalls $\Delta^{3}$-1,2-Diazetine (5).

Die in wasserfreiem Benzol gelösten Ketenderivate (I) oder (2) werden mit den ebenfalls in wasserfreiem Benzol gelösten 
$\mathrm{H}_{2} \mathrm{C}=\mathrm{C}_{\mathrm{N}}^{\stackrel{\mathrm{N}}{\mathrm{N}^{1}\left(\mathrm{R}^{1} \mathrm{R}^{2}\right)}}+\mathrm{C}_{6} \mathrm{H}_{5}-\mathrm{N}=\mathrm{N}-\mathrm{SO}_{2}-\mathrm{R} \stackrel{\text { ca. } 20^{\circ} \mathrm{C}}{\longrightarrow}$

(1)

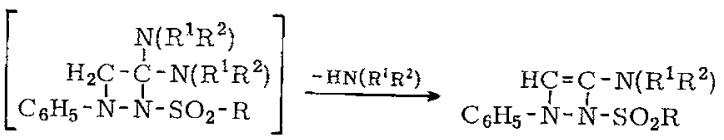

(4)

(5) ca. $20^{\circ} \mathrm{C} \uparrow-\mathrm{HOR}^{3}$
$\mathrm{HC}=\mathrm{C}-\mathrm{N}\left(\mathrm{R}^{1} \mathrm{R}^{2}\right)$ $\mathrm{RO}_{2} \mathrm{~S}-\mathrm{N}-\mathrm{N}-\mathrm{C}_{6} \mathrm{H}_{5}$

(6)
(2)

Tabelle 1. $\Delta^{3}-1,2-$ Diazetine (5) aus (1) oder (2) mit (3) in Benzol.

\begin{tabular}{|c|c|c|c|c|c|c|c|}
\hline \multicolumn{2}{|c|}{ (1) } & \multicolumn{3}{|c|}{ (2) } & \multirow[t]{2}{*}{ (3) } & \multicolumn{2}{|c|}{ (5) } \\
\hline $\mathrm{R}^{1}$ & $\mathrm{R}^{2}$ & $\mathrm{R}^{1}$ & $\mathbf{R}^{2}$ & $\mathrm{R}^{3}$ & & $\begin{array}{l}\text { Ausb. } \\
{[\%]}\end{array}$ & $F p\left[{ }^{\circ} \mathrm{C}\right]$ \\
\hline \multirow{3}{*}{\multicolumn{2}{|c|}{$\begin{array}{l}-\left(\mathrm{CH}_{2}\right)_{5}- \\
-\left(\mathrm{CH}_{2}\right)_{5}- \\
-\left(\mathrm{CH}_{2}\right)_{5}-\end{array}$}} & & & & \multirow{7}{*}{$\begin{array}{l}\mathrm{C}_{6} \mathrm{H}_{5} \\
\text { p-CH }-\mathrm{CH}_{3}-\mathrm{C}_{6} \mathrm{H}_{4} \\
\text { p-Cl- }-\mathrm{C}_{6} \mathrm{H}_{4} \\
\text { p-CH }-\mathrm{CH}_{3}-\mathrm{C}_{6} \mathrm{H}_{4} \\
\text { p-Cl- }-\mathrm{C}_{6} \mathrm{H}_{4} \\
\mathrm{C}_{6} \mathrm{H}_{5} \\
\text { p-CH }-\mathrm{CH}_{3}-\mathrm{C}_{6} \mathrm{H}_{4}\end{array}$} & 50,5 & $148-149$ \\
\hline & & & & & & 75,7 & $177-178$ \\
\hline & & & & & & 66,8 & $172-173$ \\
\hline $\mathrm{CH}_{3}$ & $\mathrm{CH}_{3}$ & & & & & 77 & $148-149$ \\
\hline \multirow[t]{3}{*}{$\mathrm{CH}_{3}$} & $\mathrm{CH}_{3}$ & & & & & 72,5 & $153-154$ \\
\hline & & $\mathrm{CH}_{3}$ & $\mathrm{CH}_{3}$ & $\mathrm{C}_{2} \mathrm{H}_{5}$ & & 60,2 & $142-144$ \\
\hline & & $\mathrm{CH}_{3}$ & $\mathrm{CH}_{3}$ & $\mathrm{C}_{2} \mathrm{H}_{5}$ & & 67 & 148 \\
\hline
\end{tabular}

$$
\text { (1) }+\mathrm{C}_{6} \mathrm{H}_{5}-\mathrm{CH}=\mathrm{N}-\mathrm{SO}_{2} \mathrm{R} \stackrel{80^{\circ} \mathrm{C}}{\longrightarrow}\left[\begin{array}{c}
\mathrm{N}\left(\mathrm{R}^{1} \mathrm{R}^{2}\right) \\
\mathrm{H}_{2} \mathrm{C}-\mathrm{C}-\mathrm{N}_{1}\left(\mathrm{R}^{1} \mathrm{R}^{2}\right) \\
\mathrm{H} \mathrm{C}-\mathrm{N}-\mathrm{SO}_{2} \mathrm{R} \\
1 \\
\mathrm{C}_{6} \mathrm{H}_{5}
\end{array}\right]
$$

$$
\begin{aligned}
& \downarrow \\
& \mathrm{HN}\left(\mathrm{R}^{1} \mathrm{R}^{2}\right)+\begin{array}{c}
\mathrm{HC}=\mathrm{C}-\mathrm{N}\left(\mathrm{R}^{1} \mathrm{R}^{2}\right) \\
\mathrm{H}-\mathrm{N}-\mathrm{SO}_{2} \mathrm{R} \\
\mathrm{C}_{6} \mathrm{H}_{5}
\end{array}
\end{aligned}
$$

die $\mathrm{N}=\mathrm{N}$-Doppelbindung für wahrscheinlicher als die Cycloaddition $\mathrm{zu}(6)$.

$\mathrm{N}$-Sulfonylimine (7) reagieren auch mit dem im Vergleich zu (1) und (2) weniger aktiven Enamin 1-Morpholinocyclohexen (10) (Molverhältnis $1: 1$ in siedendem Benzol) unter Cycloaddition zu Verbindungen der Struktur (11) (Elementaranalyse, Molekulargewicht, IR- und NMR-Spektrum). In saurer Lösung hydrolysieren die Verbindungen (11) zu den Ketonen (12).

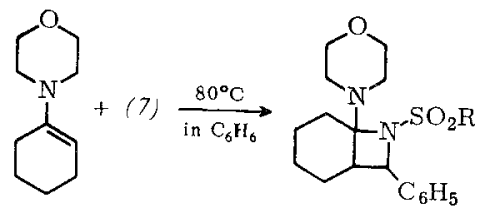

(10)

(11)

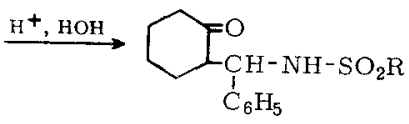

Eingegangen am 27. Januar 1966

$[Z 153]$

[1] D. H. Clemens, J. A. Bell u. J. L. O'Brien, J. org. Chemistry 29, 2932 (1964).

[2] R. Gleiter, Dissertation, Technische Hochschule Stuttgart, 1964; G. Kiefer, Diplomarbeit, Technische Hochschule Stuttgart, 1964.

[3] W. Königs, Ber. dtsch. chem. Ges. 10, 1533 (1877).

[4] R. Albrecht, G. Kresze u. B. Mlakar, Chem. Ber. 97, 483 (1964).

Tabelle 2. 2-Azetine (9) aus (1) mit (7) in Benzol bei $80^{\circ} \mathrm{C}$

\begin{tabular}{l|l|l|l}
\hline \multicolumn{1}{c|}{} & \multicolumn{1}{|c}{ (1) } & \multicolumn{2}{|c}{ (9) } \\
$\mathrm{R}^{1}+\mathrm{R}^{2}$ & $\mathrm{R}$ & $\begin{array}{l}\text { Ausb. } \\
{[\%]}\end{array}$ & $\mathrm{Fp}\left[{ }^{\circ} \mathrm{C}\right]$ \\
\hline$-\left(\mathrm{CH}_{2}\right)_{5}-$ & $\mathrm{C}_{6} \mathrm{H}_{5}$ & 68 & $132--133$ \\
$-\left(\mathrm{CH}_{2}\right)_{5}-$ & $\mathrm{p}-\mathrm{CH}_{3}-\mathrm{C}_{6} \mathrm{H}_{4}$ & 60 & $179-181$ \\
$-\left(\mathrm{CH}_{2}\right)_{5}-$ & $\mathrm{p}-\mathrm{Cl}_{-}-\mathrm{C}_{6} \mathrm{H}_{4}$ & 66,8 & $196-198$ \\
$-\left(\mathrm{CH}_{2}\right)_{2}-\mathrm{O}-\left(\mathrm{CH}_{2}\right)_{2}-$ & $\mathrm{C}_{6} \mathrm{H}_{5}$ & 35 & $146--149$ \\
$-\left(\mathrm{CH}_{2}\right)_{2}-\mathrm{O}-\left(\mathrm{CH}_{2}\right)_{2}-$ & $\mathrm{p}-\mathrm{CH}_{3}-\mathrm{C}_{6} \mathrm{H}_{4}$ & 34,3 & 166
\end{tabular}

Sulfonen (3) oder (7) im Molverhältnis 1:1 zusammengegeben und je nach Reaktionsfähigkeit des Sulfons 2 Tage bei Raumtemperatur stehen gelassen oder 2 Std. unter Rückfluß erhitzt. Nach Abdestillieren des Benzols werden die meist als Öle anfallenden Produkte durch Anreiben mit Äther oder Petroläther kristallin und können durch Umkristallisieren aus Acetonitril oder Essigester analysenrein erhalten werden. Elementaranalyse, Molekulargewichte, IR- und NMR-Spektren beweisen das Vorliegen der $\Delta^{3}$-1,2-Diazetine und 2-Azetine. Die 1:1-Addukte (4) und (8), die vermutlich primär gebildet werden, konnten unter den angewendeten Bedingungen nicht abgefangen werden.

Die Richtung der Cycloaddition mit den Sulfonyliminen (7) ist durch NMR-Untersuchungen (AB-Spektrum bei $\tau \approx 6,7$, $\mathrm{J}_{\mathrm{AB}}=17 \mathrm{~Hz}$ ) sichergestellt, während bei den Reaktionen mit (3) die Bildung der isomeren Verbindungen (6) nicht ausgeschlossen werden konnte. Die Bildung von (5) halten wir auf Grund des elektronischen Einflusses der Sulfonylgruppe auf 